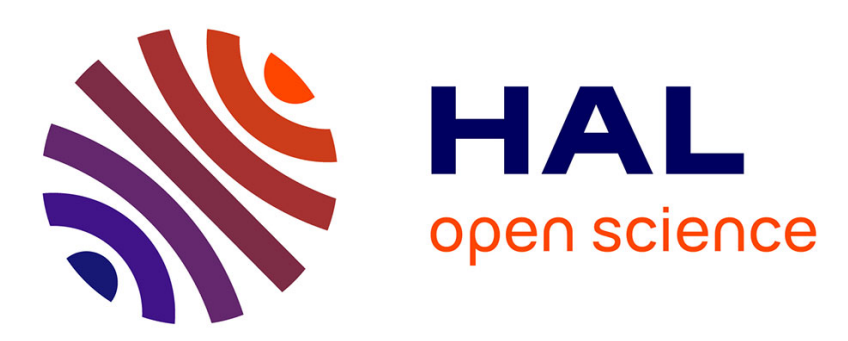

\title{
Extended orthogonal matching pursuit for robust and fast target localisation in multiple-input multiple-output radar
}

\author{
Mathieu Cattenoz, Laurent Savy, Sylvie Marcos
}

\section{- To cite this version:}

Mathieu Cattenoz, Laurent Savy, Sylvie Marcos. Extended orthogonal matching pursuit for robust and fast target localisation in multiple-input multiple-output radar. IET Radar Sonar and Navigation, 2017, 11 (11), p. 1709 - 1717. 10.1049/iet-rsn.2017.0008 . hal-01664640

\author{
HAL Id: hal-01664640 \\ https://hal.science/hal-01664640
}

Submitted on 3 Mar 2020

HAL is a multi-disciplinary open access archive for the deposit and dissemination of scientific research documents, whether they are published or not. The documents may come from teaching and research institutions in France or abroad, or from public or private research centers.
L'archive ouverte pluridisciplinaire HAL, est destinée au dépôt et à la diffusion de documents scientifiques de niveau recherche, publiés ou non, émanant des établissements d'enseignement et de recherche français ou étrangers, des laboratoires publics ou privés. 


\title{
Extended orthogonal matching pursuit for robust and fast target localisation in multiple-input multiple-output radar
}

\author{
Mathieu Cattenoz ${ }^{1, *}$, Laurent Savy ${ }^{2}$, Sylvie Marcos ${ }^{1}$ \\ ${ }^{1}$ L2S, 3 rue Joliot-Curie, 91192 Gif-sur-Yvette, France \\ ${ }^{2}$ ONERA, chemin de la Hunière, 91123 Palaiseau, France \\ "E-mail:mathieu.cattenoz@Iss.supelec.fr
}

\begin{abstract}
The multiple-input multiple-output (MIMO) radar combines ultra-wide angular illumination on transmit and narrow transmission angular resolution after processing on receive. Orthogonal waveforms are needed in theory for all independent transmitters, but this property is usually not achievable in practice, therefore leading to a degraded ambiguity function. In this study, the authors focus on phase codes waveform, which gives quasi-optimal angle and range resolution. On the other hand, they induce a harmful "floor" of high-level sidelobes in the range-angle domain. The authors propose to apply the orthogonal matching pursuit (OMP) to the MIMO radar signals. Therefore, it becomes theoretically possible to detect targets initially hidden by high-level sidelobes. Nevertheless, the authors identify phenomenon (grid granularity, neighbour influence) that lead to possible localisation error resulting in ghost targets. Considering this, the authors propose an extension of the OMP rejection step that includes, in addition to the expected target position, the neighbour positions within the uncertainty area. The authors show that this extension allows them to suppress the sensibility of the OMP to localisation error, even in case of very close targets. Finally, the authors demonstrate the performance of the proposed method on simulations as well as on experimental MIMO radar signals.
\end{abstract}

\section{Introduction}

Modern radar systems are generally based on active antennas, transmitting the signal generated by agile waveform synthesisers, and adaptively processing the received echoes, for extraction of targets from clutter and identification of potential threats. Classical radar modes involve electronic scanning of a focused beam, to successively explore the directions of interest. Modern ground radars also use a broadened beam in elevation on transmit, allowing longer illumination time. On receive, multiple simultaneous focused beams are formed by processing through digital beamforming. This technique is known as stacked beams on receive. The coherent (co-located) multiple-input multiple-output (MIMO) radar is an alternative to the last techniques [1], [2], [3], [4]. It consists of space-time coding on transmit for each individual transmitter (or sub-array), allowing to identify each of them by a convenient coherent processing on receive, and hence to recover the angular directivity of the whole antenna. The coherent processing on receive is obtained via a general matched filter in range, angle and Doppler. From a radar functional point of view, everything appears as if all directions were explored simultaneously by coded focused beams, allowing a wide-angle domain to be explored instantaneously without loss of transmit directivity on target [5]. The coherent co-located MIMO is a mean for a radar to obtain an ultra-wide angular beam on transmit (more than the classical factor 3 or 4 compared with the focused beamwidth) with good sidelobe and mainlobe properties 
(ripple, sidelobe level). Ultra-wide beams are required for instance for transitory targets (helicopter pop-up, periscope), or slow small-moving targets in competition with clutter where longer illumination times are needed for Doppler separation.

Theoretically, MIMO radar waveforms can be perfectly extracted from the received signal since supposed perfectly orthogonal with each other. This conservative hypothesis is often assumed in the literature. In practice, it is a true challenge to generate waveforms that are perfectly orthogonal, or it is at the price of notable concessions on other radar capacities, such as range resolution or transmitted power. For instance, phase codes have good properties (e.g. range resolution) in comparison with other waveforms (frequency division multiple access (FDMA), circulating waveform, etc.) [6], but the ambiguity function remains still damaged on a large domain because of the coming up of a "floor" of grating sidelobes in all range/angle/speed dimensions. This effect originates from the numerous sidelobes induced by the imperfections of the auto and cross-correlations of phase codes. Consequently, it will lead to increase the probability of false alarms, or to induce desensitisation when secondary targets are buried in the high-level sidelobes of targets of higher power.

Then it is necessary to go beyond the classical matched filter - defined so to maximize the signal-tonoise ratio (SNR) - to mitigate the processing sidelobes in MIMO radars. A solution could consist in computing the multi-target matched filter; nevertheless, its direct implementation is impractical in practice. Different approaches to approximate the global matched filter are interesting, among them: Capon and subspace-based methods, compressed sensing, etc. Moreover, MIMO radars are good candidates for adaptive localisation and detection techniques [7], which are known to have much better resolution and much better interference rejection capability than their data-independent counterparts.

In this paper, we will first exhibit the impact of matched filter on non-perfectly orthogonal waveforms, in both formal and visual points of view. Afterwards, taking into account radar operational constraints (e.g. size of available data, computation capacities), we will introduce the Orthogonal Matching Pursuit (OMP) method where each target is detected and its contribution removed from the ambiguity function, successively. The limitation of this method with respect to localisation error will be analysed, in particular concerning the coming up of "ghost targets". In order to compensate this issue, we will propose an extension for the rejection step of the OMP algorithm, defined so to cope with both the grid problem and the issue of closely spaced targets. The robustness and performance of the proposed extension will be tested on simulated MIMO radar signals and compared with the classical OMP. Finally, we will exploit real data from experiments on a MIMO-capable radar testbed in order to show the performance of the introduced method in situation of signal model mismatch.

\section{Limitations of the classical methods applied to MIMO radar waveforms}

\subsection{Signal modeling for coherent MIMO radar waveforms}

We introduce here a formal writing in the context of realistic - i.e. non-perfectly orthogonal - MIMO radar waveforms, and allowing to describe the adapted filter as well other methods of higher complexity. In this paper, we consider that the MIMO radar transmits a train of identical pulses, and that each antenna transmits its specific intrapulse code during these pulses. In the context of coherent co-located MIMO radar, all elementary antennas transmit distinct waveforms. The signal during the pulse is assumed discrete, and $K$ is the number of samples. The vector $\mathbf{w}_{m}$ of size $K \times 1$ denotes the waveform, in time domain, assigned to the $m^{\text {th }}$ antenna, among a total of $M$ antennas. Thus, the signal $\mathbf{r}_{T}$ of size $K \times 1$ transmitted by the array is

$$
\mathbf{r}_{T}(\theta)=\sum_{m=1}^{M} g_{m}(\theta) e^{j \mathbf{x}_{m}^{T} \mathbf{k}(\theta)} \mathbf{w}_{m}
$$


where $\theta$ is the considered direction, $g_{m}(\theta)$ is the gain of transmitting antenna $m$ in direction $\theta, \mathbf{x}_{m}$ is the position vector of antenna $m, \mathbf{k}(\theta)$ is the wave vector. The notation $(\cdot)^{T}$ represents the matrix transpose.

Concatenating all transmitted waveforms $\mathbf{w}_{m}$ in a single matrix $\mathbf{W}=\left[\mathbf{w}_{1} \cdots \mathbf{w}_{M}\right]$ of size $K \times M$, we get

$$
\mathbf{r}_{T}(\theta)=\mathbf{W} \mathbf{s}_{T}(\theta)
$$

where the vector $\mathbf{s}_{T}(\theta)$ of size $M \times 1$ is the steering vector for transmission (Tx) whose components are given by

$$
\left(\mathbf{s}_{T}(\theta)\right)_{m}=g_{m}(\theta) e^{j \mathbf{x}_{m}^{T} \mathbf{k}(\theta)}
$$

with $(\cdot)_{m}$ denoting the $m^{\text {th }}$ element of a vector.

We will now write the expression in the spectrum domain, by considering $\tilde{\mathbf{v}}$ the spectrum of any time vector $\mathbf{v}$, and $\tilde{\mathbf{W}}=\left[\tilde{\mathbf{w}}_{1} \cdots \tilde{\mathbf{w}}_{M}\right]$

$$
\tilde{\mathbf{r}}_{T}(\theta)=\tilde{\mathbf{W}} \mathbf{s}_{T}(\theta)
$$

For a target, the range, the Doppler speed, and the angle of view can be detected. For the sake of simplicity, we will make two considerations in the following:

- One single antenna on receive. Indeed, it has been demonstrated that transmission and reception processing schemes are completely decoupled for MIMO radars [8].

- No consideration of the Doppler dimension, in order to work in the range/angle domain only. As an illustration, it corresponds to a situation of detection of slow moving targets only.

If a target of unit amplitude is present in direction $\theta_{t}$ at range $r_{t}$, then the signal $\tilde{\mathbf{r}}_{R}$ of size $K \times 1$ received on the antenna is (with $\odot$ denoting the Hadamard product)

$$
\tilde{\mathbf{r}}_{R}=\tilde{\mathbf{W}} \mathbf{s}_{T}\left(\theta_{t}\right) \odot \tilde{\mathbf{s}}_{f}\left(r_{t}\right)
$$

where the vector $\tilde{\mathbf{s}}_{f}(r)$ of size $K \times 1$ is the range-"steering" vector whose components are

$$
\left(\tilde{\mathbf{s}}_{f}(r)\right)_{k}=e^{i 2 \pi k \frac{2 r}{c} \Delta f}
$$

with $\Delta f$ the frequency sampling interval and $c$ the wave propagation speed. In other words, the effect of $\tilde{\mathbf{s}}_{f}(r)$ in time domain is time-shifting the waveforms by the round-trip delay $2 r / c$.

The first step of the optimal coherent MIMO radar processing on receive is the transmitter separation through the correlations of the received signal with the transmitted waveforms, one by one. In the spectral domain, this operation consists in multiplying the received signal spectrum with each waveform spectrum. Let us explicit the expression $\tilde{\mathbf{a}}_{m}\left(r_{t}, \theta_{t}\right)$ of the signal of size $K \times 1$ after correlation with waveform $m$, in the spectral domain (with $(\cdot)^{*}$ denoting the conjugate)

$$
\begin{aligned}
\tilde{\mathbf{a}}_{m}\left(r_{t}, \theta_{t}\right) & =\sum_{n=1}^{M}\left(\mathbf{s}_{T}\left(\theta_{t}\right)\right)_{n} \cdot \tilde{\mathbf{s}}_{f}\left(r_{t}\right) \odot \tilde{\mathbf{w}}_{n} \odot \tilde{\mathbf{w}}_{m}^{*} \\
& =\tilde{\mathbf{W}} \mathbf{s}_{T}\left(\theta_{t}\right) \odot \tilde{\mathbf{s}}_{f}\left(r_{t}\right) \odot \tilde{\mathbf{w}}_{m}^{*}
\end{aligned}
$$

Finally, the expression of vector $\tilde{\mathbf{a}}\left(r_{t}, \theta_{t}\right)$ of the received data after pulse compression (whose size is $K M \times 1)$ is written

$$
\tilde{\mathbf{a}}\left(r_{t}, \theta_{t}\right)=\left[\begin{array}{c}
\tilde{\mathbf{a}}_{1}\left(r_{t}, \theta_{t}\right) \\
\vdots \\
\tilde{\mathbf{a}}_{M}\left(r_{t}, \theta_{t}\right)
\end{array}\right]
$$


Considering now $\alpha_{t}$ the complex amplitude of the target and $\mathbf{n}$ the additive noise, we can express the received vector after pulse compression $\tilde{\mathbf{z}}$ in the spectral domain

$$
\tilde{\mathbf{z}}=\alpha_{t} \tilde{\mathbf{a}}\left(r_{t}, \theta_{t}\right)+\tilde{\mathbf{n}}
$$

\subsection{Sidelobes issue with the matched filter}

Once the transmitter separation is done, the matched filter on receive consists in range compression and beamforming on transmit (and also on receive in the case of multiple reception antennas). The estimation of $\alpha_{t}$ for all $(r, \theta)$ hypotheses can be expressed the following way [ $\otimes$ denoting the Kronecker product and $(\cdot)^{H}$ denoting the conjugate transpose]

$$
\hat{\alpha}(r, \theta)=\frac{\left[\mathbf{s}_{T}(\theta) \otimes \tilde{\mathbf{s}}_{f}(r)\right]^{H} \tilde{\mathbf{z}}}{\left\|\mathbf{s}_{T}(\theta) \otimes \tilde{\mathbf{s}}_{f}(r)\right\|^{2}}
$$

We isolate the term that depends on the waveforms $\tilde{\mathbf{w}}_{m}$

$$
F\left(\theta, \theta_{t}, r, r_{t}\right) \triangleq\left[\mathbf{S}_{T}(\theta) \otimes \tilde{\mathbf{s}}_{f}(r)\right]^{H} \tilde{\mathbf{a}}\left(r_{t}, \theta_{t}\right)
$$

After several developments, we get

$$
\begin{aligned}
F\left(\theta, \theta_{t}, r, r_{t}\right)= & {\left[\tilde{\mathbf{s}}_{f}(r) \odot \tilde{\mathbf{s}}_{f}^{*}\left(r_{t}\right)\right]^{H}\left[\sum_{m=1}^{M}\left(\mathbf{s}_{T}^{*}(\theta)\right)_{m}\left(\mathbf{s}_{T}\left(\theta_{t}\right)\right)_{m} \tilde{\mathbf{w}}_{m} \odot \tilde{\mathbf{w}}_{m}^{*}\right.} \\
& \left.+\sum_{m=1}^{M} \sum_{n \neq m}\left(\mathbf{s}_{T}^{*}(\theta)\right)_{m}\left(\mathbf{s}_{T}\left(\theta_{t}\right)\right)_{n} \tilde{\mathbf{w}}_{n} \odot \tilde{\mathbf{w}}_{m}^{*}\right]
\end{aligned}
$$

It is worth noting that:

- $\tilde{\mathbf{w}}_{m} \odot \tilde{\mathbf{w}}_{m}^{*}$ is the autocorrelation of waveform $m$ in the spectral domain. In the case of hypothetical perfectly orthogonal waveforms, it is a constant vector (i.e. a Dirac function in the time domain).

- $\tilde{\mathbf{w}}_{n} \odot \tilde{\mathbf{w}}_{m}^{*}$ with $n \neq m$ is the cross-correlation function of waveform $n$ with waveform $m$ in the spectral domain. In the case of hypothetical perfectly orthogonal waveforms, it is a zero-value vector.

In the case of a hypothetical family of perfectly orthogonal waveforms, expression (13) becomes

$$
F_{\text {th }}\left(\theta, \theta_{t}, r, r_{t}\right)=\tilde{\mathbf{s}}_{f}^{H}(r) \tilde{\mathbf{s}}_{f}\left(r_{t}\right) \cdot \mathbf{s}_{T}^{H}(\theta) \mathbf{s}_{T}\left(\theta_{t}\right)
$$

This is actually the ideal "interference figure" response (two sinc profiles in both $r$ and $\theta$ directions) coming from the sum of vector components (actually $\left\|\mathbf{s}_{T}(\theta) \otimes \tilde{\mathbf{s}}_{f}(r)\right\|^{2}$ ) and due to the limited bandwidth and spatial resolution. We precise that this interference figure is not included in what we call sidelobes in this work. Consequently, in the situation of perfect waveform orthogonality, it comes out of (14) that no sidelobes appear.

In the realistic situation of non-perfectly orthogonal waveforms, the sums of auto and cross-correlation functions in expression (13) induce residues in the ambiguity function, which are at the root of the grating sidelobes all around the mainlobe of the target.

We have mentioned that the high-level sidelobes may be above the noise level, and consequently, they can induce false alarms. Moreover, the sidelobes of a target are likely to hide secondary targets of lower power. In order to exhibit the coming out of sidelobes in the case of non-perfectly orthogonal waveforms and make the comparison with the ideal orthogonal case, we process a simulation with the generation of 


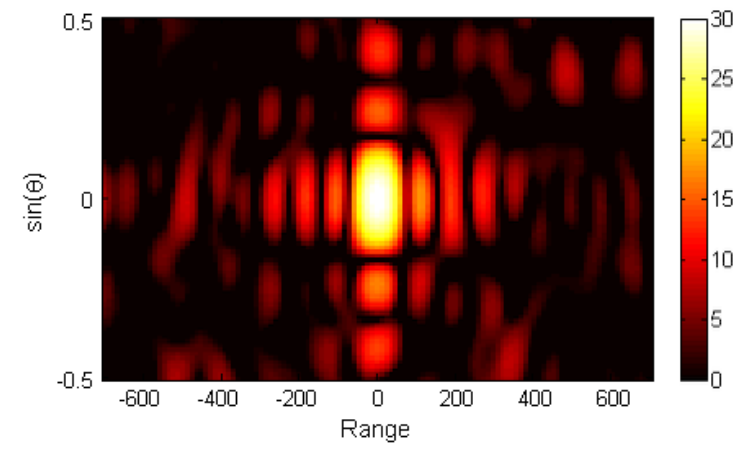

(a)

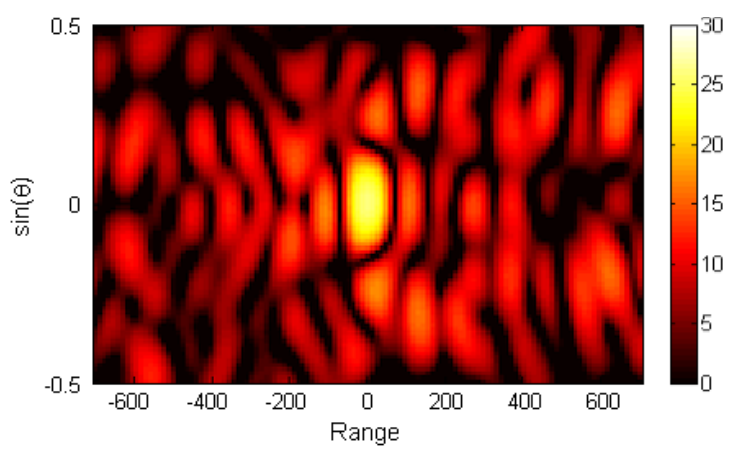

(b)

Fig. 1. Target amplitude estimation based on matched filter for one point target

a Simulation of a family of perfectly orthogonal waveforms

b Simulation of a family of Gold codes [9]

a Gold [9] code family of 127 chips. Indeed, these phase codes designed for digital communications have very good auto and cross-correlation properties, but are still not perfectly orthogonal. Here, the waveforms are first generated and added with Gaussian noise, then sampled, filtered, modulated, summed, and time delayed on target. In the present case, the target is placed at the origin (range $r_{t}=0$, angle $\theta_{t}=0$ ). Then, we perform a demodulation and a decimation of the signals, and eventually a matched filter for each waveform. Here is a list of the relevant parameters of the simulations:

- Output (i.e. after processing) SNR: $30 \mathrm{~dB}$;

- Number of transmitters: $M=12$;

- Number of code chips: $K=127$.

We have set the parameters so to give (through noise power normalisation after processing) a noise level around $0 \mathrm{~dB}$ and a target peak value similar to the expected output SNR.

The resulting output for an ideal hypothetic ambiguity function [based on (14)] is displayed on Fig. 1(a), We clearly notice the intersection of the range and angle sinc profiles originating from the point target (i.e. the "interfering figure"). As expected, the main lobe is located in the center and reaches a value close to 30 $\mathrm{dB}$. All around appears the "floor" of noise at around $0 \mathrm{~dB}$. This figure has to be compared with the resulting output for the simulated realistic signals given on Fig. 1(b). For this ambiguity function, the main lobe is also in the centre and reaches a value close to $30 \mathrm{~dB}$ (around $5 \mathrm{~dB}$ below though). To the difference with the previous situation, there is here a distinct "floor" of sidelobes distributed in all angle and range positions, with maximum power up to $10 \mathrm{~dB}$ below the peak value.

Therefore, the comparison of the two figures clearly shows that the sidelobes are indeed caused by the lack of orthogonality of the radar waveforms that induces residues when the waveform auto and crosscorrelations are added.

The main impact of the high-level sidelobes is the desensitisation to secondary targets. Indeed, targets of power above the noise level but $10 \mathrm{~dB}$ (or more) below the primary target would not be detectable in such situation, since the detection threshold should be set above the sidelobes which reach $20 \mathrm{~dB}$ here. It results in performance degradation on target detection capacities. Another impact of the sidelobes is the loss of target peak value. The loss of $5 \mathrm{~dB}$ of the target mainlobe is due to the fact that more energy is spread in the sidelobes. This has also an impact on performance degradation on target detection capacities.

Note that a reduction of the sidelobe level can be achieved - but usually at unrealistic price - by using a wider bandwidth or longer pulses (i.e. longer code sequences). 


\subsection{Adaptive methods applied to MIMO radar waveforms}

Several adaptive methods have been already studied in [10] and [11] in the framework of non-perfectly orthogonal MIMO radar waveforms. It has been demonstrated that the methods (e.g. Capon) based on a covariance matrix are usually not suitable in practice because of the difficulty to have enough data as snapshots with respect to the great dimension of $\tilde{\mathbf{z}}$. On the contrary, the Iterative Adaptive Approach (IAA) technique [12] [13] provides accurate high-resolution estimates, even with one unique snapshot. Nevertheless, it has been experienced that this method is not suitable in most practical cases because of its high computational complexity. Therefore, in this paper, we will sometimes display the IAA output but only as a reference for performance comparison with the methods introduced lately, especially in situations where the original target scene is not precisely known (experimental signals typically).

Here is the expression of the iterative estimation process at step $n$

$$
\begin{aligned}
& \hat{\alpha}_{n}\left(r_{i}, \theta_{j}\right)=\frac{\tilde{\mathbf{a}}^{H}\left(r_{i}, \theta_{j}\right) \boldsymbol{\Gamma}_{n-1}^{-1} \tilde{\mathbf{z}}}{\tilde{\mathbf{a}}^{H}\left(r_{i}, \theta_{j}\right) \boldsymbol{\Gamma}_{n-1}^{-1} \tilde{\mathbf{a}}\left(r_{i}, \theta_{j}\right)} \\
& \boldsymbol{\Gamma}_{n}=\sum_{i, j}\left|\hat{\alpha}_{n}\left(r_{i}, \theta_{j}\right)\right|^{2} \tilde{\mathbf{a}}\left(r_{i}, \theta_{j}\right) \tilde{\mathbf{a}}^{H}\left(r_{i}, \theta_{j}\right)
\end{aligned}
$$

with $\boldsymbol{\Gamma}_{0}$ initialized to the identity matrix $\mathbf{I}$ and $\left(r_{i}, \theta_{j}\right)$ taking the $(r, \theta)$ values of the parameter grid.

\section{Classical OMP applied to MIMO radar waveforms}

\subsection{Fast method for target detection and rejection}

Most radar scenes are sparse in the sense that only a small number of the range-angle-Doppler cells are occupied by objects of interest. This sparsity suggests one to approach the MIMO radar problem using the framework of compressed sensing [14][15]. In the following, we will then consider iterative algorithms of the literature issued or in close relation with the compressed sensing approach.

The OMP consists in building an approximation of the multitarget matched filter. With the Matching Pursuit (MP) algorithm, the measured signal is decomposed into the contributions of several waveforms selected from a predefined dictionary [16]. The OMP that we use here [17] can be considered as inspired by the CLEAN algorithm initially proposed in [18] [19] for deconvolution problems in radio astronomy. Note that this algorithm was later analysed as a compressed sensing imaging technique for radio interferometry [20].

The OMP algorithm is an iterative implementation of the point multitarget matched filter that consists in detecting and rejecting each contribution - and the associated sidelobes thus - of the strong targets, successively. It is initialized with $\tilde{\mathbf{z}}_{1}=\tilde{\mathbf{z}}$ and then calculated the following way for $k^{\text {th }}$ iteration

$$
\begin{aligned}
& \text { Detection step: } \text { find }\left(r_{k}, \theta_{k}\right)=\arg \max _{r, \theta} \frac{\left|\tilde{\mathbf{a}}(r, \theta)^{H} \tilde{\mathbf{z}}_{k}\right|^{2}}{\tilde{\mathbf{a}}(r, \theta)^{H} \tilde{\mathbf{a}}(r, \theta)} \\
& \text { Rejection step: } \tilde{\mathbf{z}}_{k+1}=\left(\mathbf{I}-\tilde{\mathbf{A}}_{k}\left(\tilde{\mathbf{A}}_{k}^{H} \tilde{\mathbf{A}}_{k}\right)^{-1} \tilde{\mathbf{A}}_{k}^{H}\right) \tilde{\mathbf{z}} \\
& \qquad \text { where } \tilde{\mathbf{A}}_{k}=\left[\tilde{\mathbf{A}}_{k-1}, \tilde{\mathbf{a}}\left(r_{k}, \theta_{k}\right)\right]=\left[\tilde{\mathbf{a}}\left(r_{1}, \theta_{1}\right), \cdots, \tilde{\mathbf{a}}\left(r_{k}, \theta_{k}\right)\right]
\end{aligned}
$$

At each iteration, the predominant value of the matched filter output is detected and the corresponding localisation is attributed to the newly detected target. Then, this localisation is "filtered out" from the target parameter space. Therefore, all contributions of the detected target in the output of the matched filter are 
removed, and it makes it possible then to detect the targets of lower power, which were possibly "buried" in the sidelobes of the original predominant targets. Finally, once a certain stopping criterion is satisfied e.g. related to a specific false alarm rate, the algorithm stops. In the following, we will consider a stopping threshold at $10 \mathrm{~dB}$ (after processing) over noise level, which corresponds for Gaussian noise to a probability of false alarm of $4.5 \times 10^{-5}$.

In order to compare the computational complexities between OMP and IAA, we first have identified the complexity of each operation. For all $N_{I}$ iterations of the IAA, the predominant operations are:

- Inversion of matrix $\Gamma_{0}$ of size $M K \times M K: O\left(N_{I}(M K)^{3}\right)$;

- Operations on terms of matrix of size $M K \times M K$ for all $P_{I}$ nodes of the grid: $O\left(N_{I} P_{I}(M K)^{2}\right)$.

For OMP with $N_{O}$ target detections (assuming that $N_{O} \ll M K$ ), the predominant operations are:

- Multiplication of $\tilde{\mathbf{A}}_{k}$ of size $M K \times N_{O}$ with $\left(\tilde{\mathbf{A}}_{k}^{H} \tilde{\mathbf{A}}_{k}\right)^{-1} \tilde{\mathbf{A}}_{k}^{H}$ of size $N_{O} \times M K: O\left(\left(N_{O} M K\right)^{2}\right)$;

- Scalar products of vectors of length $M K$ for all $P_{O}$ nodes of the grid: $O\left(N_{O} P_{O} M K\right)$.

Finally, the preponderant complexity of the IAA in $O\left((M K)^{2} N_{I}\left(M K+P_{I}\right)\right)$ should be compared with the one of the OMP in $O\left((M K) N_{O}\left(N_{O} M K+P_{O}\right)\right)$. In situations where the number of detected targets is small and $N_{I}$ (respectively, $P_{I}$ ) is of same order of magnitude as $N_{O}$ (respectively, $P_{O}$ ), it can be deduced that the computational complexity of the OMP is, at first order, $M K$ times smaller than the one of the (non-optimised) IAA.

In our specific context of computation on real signals, $P_{I}=4 P_{O}=3200, N_{I} \leq 10, N_{O} \leq 10$, and $M K \approx 1500$. With a processor Intel Core i3 at $1.8 \mathrm{GHz}$, around four days were needed for the IAA - nonoptimised version of the IAA though - while it was only around 5 min with the OMP, i.e. approximately $10^{3}$ times less.

\subsection{Biased target localisation issue}

The target scanning grid used for target detection is a discretisation of the target parameter space $(r, \theta)$, where each node represents a specific parameters hypothesis. During the detection step, the grid node corresponding to the highest value is attributed to a target. It is likely to be a biased localisation for this target for two reasons:

Grid granularity: It is unlikely that the maximum of the continuous ambiguity function corresponds exactly to a node of the discrete scanning grid. Therefore, a nearby node will be attributed by the algorithm as estimated localisation for the detected target. A possible way to mitigate this issue is to increase the density of the scanning grid, at the price of a proportional increase of the computational complexity.

Target proximity: When two (or more) targets are close, their powers are likely to add up in a way to create a unique maximum between the targets (due to the fact that the matched filter is only optimal for one single target). It results in the algorithm detecting only one maximum at the output of the classical matched filter and an inaccurate target localisation as well. By the way, increasing the grid density has no impact on this issue. Nonetheless, it is possible to determine the limit of extension of such influence between targets, related to a "minimum resolvable distance" (similar to Rayleigh criterion in optics) usually equal to the width of the resolution cell.

In classical OMP, a biased localisation is likely to produce residues after target rejection that could induce additional detection of "ghost" targets. It is therefore of crucial importance to give accurate estimations of the target localisations, especially the ones of the targets of strong power. 


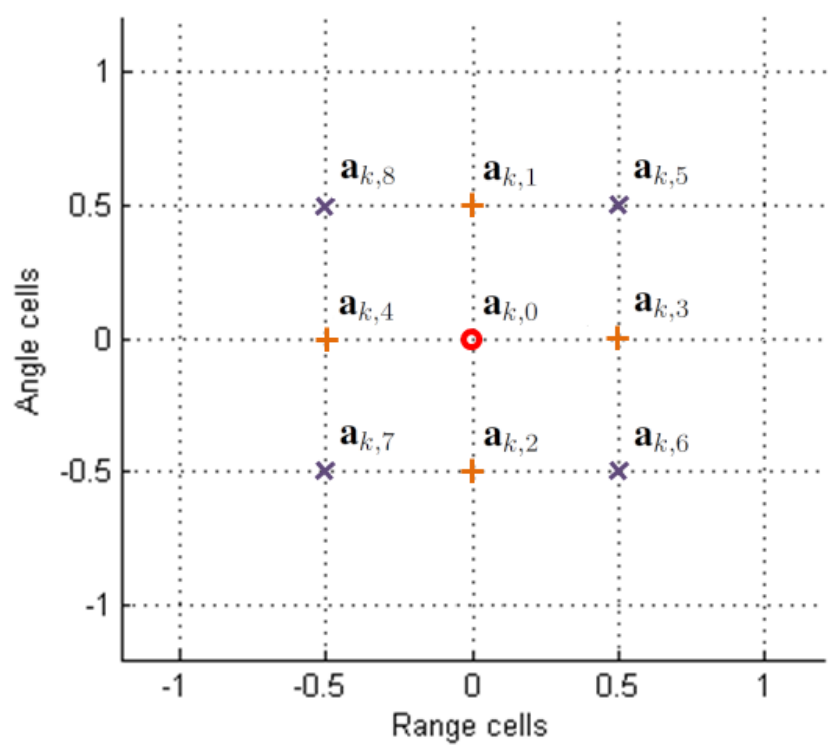

Fig. 2. Localisation on target grid nodes of the $k^{\text {th }}$ detected target and its direct neighbours

\section{Extended OMP as robustification to localisation error}

As possible improvement to localisation error issue, it is proposed in [21] to process at each iteration a joint estimation of the target states in the maximum-likelihood sense. Nevertheless, this method, as well as peak fitting methods, does not resolve the issue of very close targets. According to the authors' knowledge, no method in the literature addresses this point correctly while preserving relatively low computational load. Here, we address the radar detection, and this is a key point, because of the number of hypotheses to be tested. We seek for a "coarse" approach that is necessary and sufficient for detection.

Since the rejection area with the classical OMP is not large enough to cope with the scanning grid granularity and the influence of neighbour targets, we introduce a way to extend this area by taking into account the error between the real target localisation and the estimated one. Hence, the extension of the classical OMP that we propose consists, at each rejection step, in concatenating into the matrix $\tilde{\mathbf{A}}_{k}$ the vectors that allow one to approximate the real target vector.

For that purpose, we start with the development in Taylor series at second order of the steering vector $\tilde{\mathbf{a}}(r, \theta)$ around a grid node $\left(r_{k}, \theta_{k}\right)$

$$
\begin{aligned}
\tilde{\mathbf{a}}(r, \theta) \approx & \tilde{\mathbf{a}}\left(r_{k}, \theta_{k}\right)+\left.\left(r-r_{k}\right) \frac{\partial \tilde{\mathbf{a}}(r, \theta)}{\partial r}\right|_{r=r_{k}}+\left.\left(\theta-\theta_{k}\right) \frac{\partial \tilde{\mathbf{a}}(r, \theta)}{\partial \theta}\right|_{\theta=\theta_{k}} \\
& +\left.\frac{1}{2}\left(r-r_{k}\right)^{2} \frac{\partial^{2} \tilde{\mathbf{a}}(r, \theta)}{\partial r^{2}}\right|_{r=r_{k}}+\left.\frac{1}{2}\left(\theta-\theta_{k}\right)^{2} \frac{\partial^{2} \tilde{\mathbf{a}}(r, \theta)}{\partial \theta^{2}}\right|_{\theta=\theta_{k}} \\
& +\left.\left(r-r_{k}\right)\left(\theta-\theta_{k}\right) \frac{\partial^{2} \tilde{\mathbf{a}}(r, \theta)}{\partial r \partial \theta}\right|_{r=r_{k}, \theta=\theta_{k}}
\end{aligned}
$$

The second-order approximation in (20) shows that around a grid node, the space spanned by all the possible target locations is only defined by six vectors, corresponding to the values of the function $\tilde{\mathbf{a}}(r, \theta)$ and of its first- and second-order derivatives taken at the grid nodes.

The objective then is to approximate the terms of the development in Taylor series with finite differences, derived from a target grid oversampled only by a factor of 2 . Hence, we consider a target grid made of two 
nodes per cell dimension, with $\Delta r$ and $\Delta \theta$ the resolution cell widths for range and Tx angle, respectively. Typically, $\Delta r=c / 2 B$ and $\Delta \theta=\arcsin (\lambda / L)$, with $c$ the wave propagation speed, $B$ the transmission bandwidth, $\lambda$ the transmission wavelength and $L$ the whole radar antenna length. Then, we introduce the direct neighbour vectors, whose localisations are represented on Fig. 2

$$
\begin{array}{ll}
\mathbf{a}_{k, 0}=\tilde{\mathbf{a}}\left(r_{k}, \theta_{k}\right) & \mathbf{a}_{k, 5}=\tilde{\mathbf{a}}\left(r_{k}+\Delta r / 2, \theta_{k}+\Delta \theta / 2\right) \\
\mathbf{a}_{k, 1}=\tilde{\mathbf{a}}\left(r_{k}, \theta_{k}+\Delta \theta / 2\right) & \mathbf{a}_{k, 6}=\tilde{\mathbf{a}}\left(r_{k}+\Delta r / 2, \theta_{k}-\Delta \theta / 2\right) \\
\mathbf{a}_{k, 2}=\tilde{\mathbf{a}}\left(r_{k}, \theta_{k}-\Delta \theta / 2\right) & \mathbf{a}_{k, 7}=\tilde{\mathbf{a}}\left(r_{k}-\Delta r / 2, \theta_{k}-\Delta \theta / 2\right) \\
\mathbf{a}_{k, 3}=\tilde{\mathbf{a}}\left(r_{k}+\Delta r / 2, \theta_{k}\right) & \mathbf{a}_{k, 8}=\tilde{\mathbf{a}}\left(r_{k}-\Delta r / 2, \theta_{k}+\Delta \theta / 2\right) \\
\mathbf{a}_{k, 4}=\tilde{\mathbf{a}}\left(r_{k}-\Delta r / 2, \theta_{k}\right) &
\end{array}
$$

The objective is to approximate the terms of the development in Taylor series with these specific vectors, taken directly from the target grid

$$
\begin{array}{ll}
\mathbf{c}_{k, 0}=\mathbf{a}_{k, 0} & \text { term of order } 0 \\
\mathbf{c}_{k, 1}=\mathbf{a}_{k, 3}-\mathbf{a}_{k, 4} & \\
\mathbf{c}_{k, 2}=\mathbf{a}_{k, 1}-\mathbf{a}_{k, 2} & \\
\mathbf{c}_{k, 3}=\mathbf{a}_{k, 3}+\mathbf{a}_{k, 4}-2 \mathbf{a}_{k, 0} & \\
\mathbf{c}_{k, 4}=\mathbf{a}_{k, 1}+\mathbf{a}_{k, 2}-2 \mathbf{a}_{k, 0} & \text { crorms of first order } \\
\mathbf{c}_{k, 9}=\left(\mathbf{a}_{k, 5}-\mathbf{a}_{k, 6}\right)-\left(\mathbf{a}_{k, 8}-\mathbf{a}_{k, 7}\right) & \text { cross term of second order }
\end{array}
$$

Note that it is not necessary to take the multiplicative scalars into account since it is in a purpose of rejection of the entire vector. Note also that first-order derivatives are the classical "delta" channels used in radar to improve measurements accuracy.

In theory, the-second order approximation accuracy, and hence the subspace spanned by the derivatives vectors, shall be independent to any change of variables in the function $\tilde{\mathbf{a}}(r, \theta)$ of (20). Actually, it appeared that it was not the case, for the reason that we approximate here the derivatives by coarse finite differences - which can be easily computed. After some trials, we found that the best estimation with coarse halfcell finite differences of the subspace spanned by the true derivatives in 20) is obtained by using also the derivatives computed after a rotation of $45^{\circ}$ of the parameter plane

$$
\begin{array}{ll}
\mathbf{c}_{k, 5}=\mathbf{a}_{k, 5}-\mathbf{a}_{k, 7} & \\
\mathbf{c}_{k, 6}=\mathbf{a}_{k, 8}-\mathbf{a}_{k, 6} & \\
\mathbf{c}_{k, 7}=\mathbf{a}_{k, 5}+\mathbf{a}_{k, 7}-2 \mathbf{a}_{k, 0} & \\
\mathbf{c}_{k, 8}=\mathbf{a}_{k, 8}+\mathbf{a}_{k, 6}-2 \mathbf{a}_{k, 0} & \text { cross term of second order } \\
\mathbf{c}_{k, 10}=\left(\mathbf{a}_{k, 1}-\mathbf{a}_{k, 3}\right)-\left(\mathbf{a}_{k, 4}-\mathbf{a}_{k, 2}\right) & \text { crost order }
\end{array}
$$

Since $\mathbf{c}_{k, 9}$ can be computed by $\left(\mathbf{c}_{k, 7}-\mathbf{c}_{k, 8}\right)$, and $\mathbf{c}_{k, 10}$ by $\left(\mathbf{c}_{k, 4}-\mathbf{c}_{k, 3}\right)$, these two terms are unnecessary. Finally, the nine remaining vectors $\left(\mathbf{c}_{k, i}\right)_{i=0 \cdots 8}$ are concatenated into the matrix $\tilde{\mathbf{A}}_{k}$ to extend the OMP rejection area

$$
\tilde{\mathbf{A}}_{k}=\left[\tilde{\mathbf{A}}_{k-1}, \mathbf{c}_{k, 0}, \mathbf{c}_{k, 1}, \mathbf{c}_{k, 2}, \mathbf{c}_{k, 3}, \mathbf{c}_{k, 4}, \mathbf{c}_{k, 5}, \mathbf{c}_{k, 6}, \mathbf{c}_{k, 7}, \mathbf{c}_{k, 8}\right]
$$

Moreover, it is worth noting that the vectors $\left(\mathbf{c}_{k, i}\right)_{i=0 \cdots 8}$ are derived from a linear invertible combination of the steering vectors $\left(\mathbf{a}_{k, i}\right)_{i=0 \cdots 8}$ (whose localisations are represented on Fig. 2). Therefore, it demonstrates that it is identical to express the $\tilde{\mathbf{A}}_{k}$ matrix directly with the steering vectors of the estimated target plus its eight direct neighbours, i.e. $\left(\mathbf{a}_{k, i}\right)_{i=0 \cdots 8}$ instead of $\left(\mathbf{c}_{k, i}\right)_{i=0 \cdots 8}$

$$
\tilde{\mathbf{A}}_{k}=\left[\tilde{\mathbf{A}}_{k-1}, \mathbf{a}_{k, 0}, \mathbf{a}_{k, 1}, \mathbf{a}_{k, 2}, \mathbf{a}_{k, 3}, \mathbf{a}_{k, 4}, \mathbf{a}_{k, 5}, \mathbf{a}_{k, 6}, \mathbf{a}_{k, 7}, \mathbf{a}_{k, 8}\right]
$$




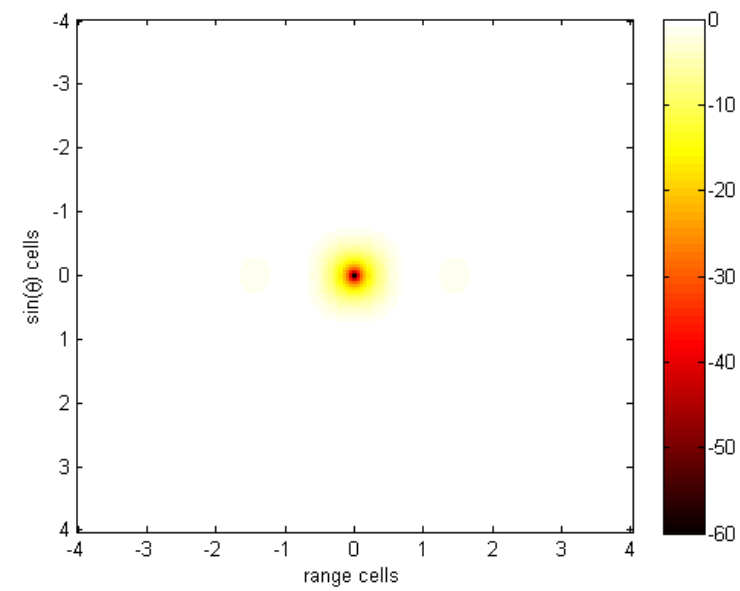

(a)

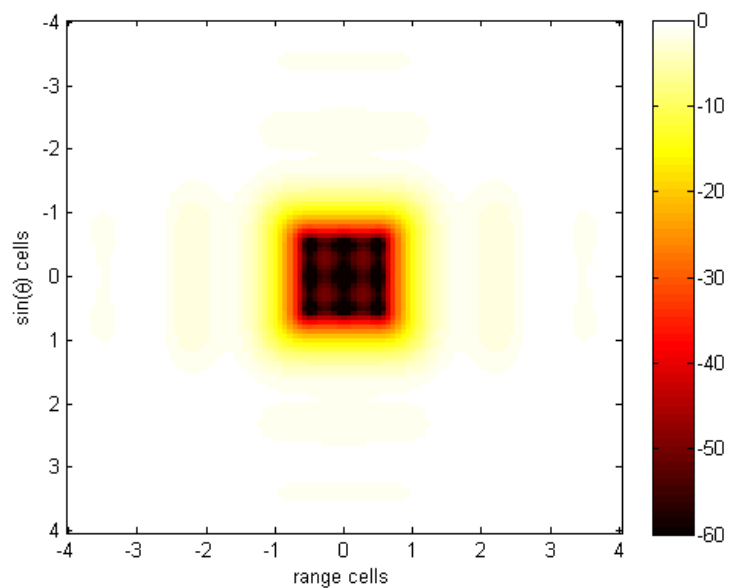

(b)

Fig. 3. Power gain in target parameter space after one rejection a Classical OMP rejection

b Extended OMP rejection

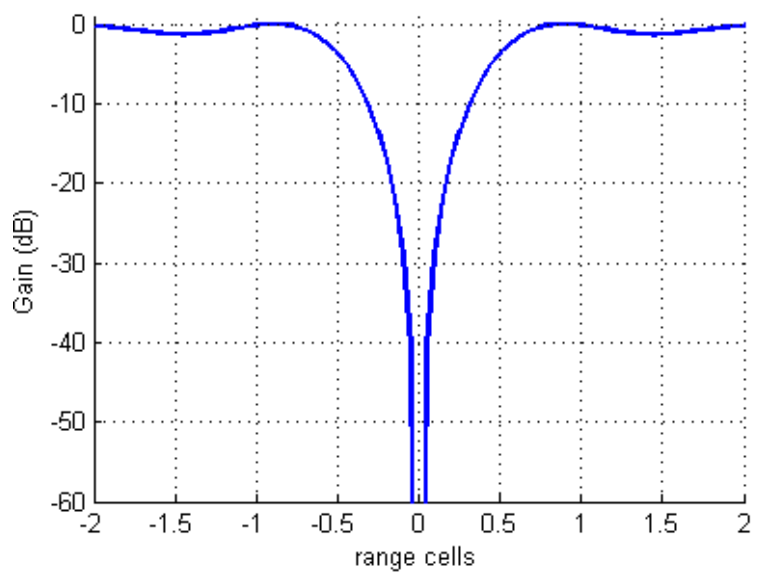

(a)

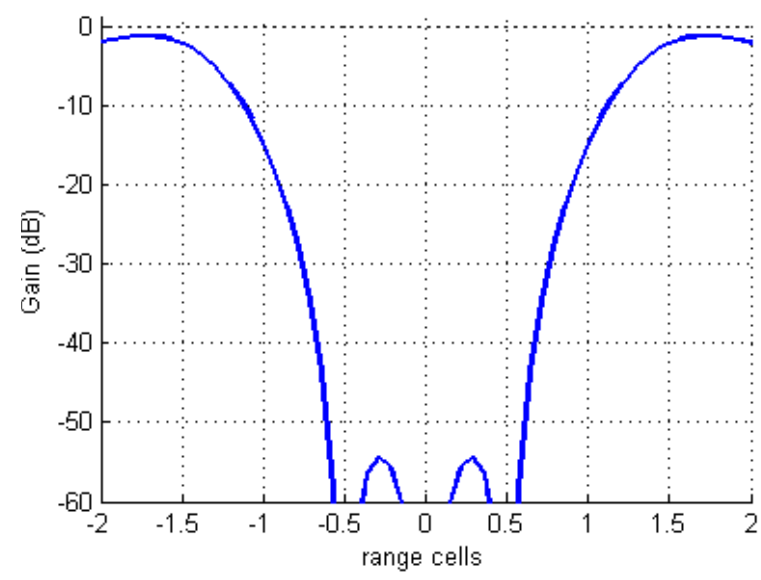

(b)

Fig. 4. Range cut of power gain in target parameter space after one rejection a Classical OMP rejection b Extended OMP rejection

The effects of the classical and the extended rejection on the power gain of the rejection step in target parameter space are displayed on Fig. 3. It can be noticed that the extended rejection mitigates the complete cell, to the difference with the classical rejection which mitigates only a reduced area around the considered localisation. The gain profiles are more clearly displayed for the range cuts at target angle on Fig. 4.

Finally, the method of robustification proposed here consists in rejecting not only the estimated - and possibly biased - position of the detected target, but also the other possible positions within a resolution cell span for both localisation parameters; and the interest of the proposed extension is that the filtered area actually covers the area of localisation uncertainty due to both the scanning grid granularity and the influence of neighbour targets. On the other hand, note that this method is at the price of a loss in resolution. 


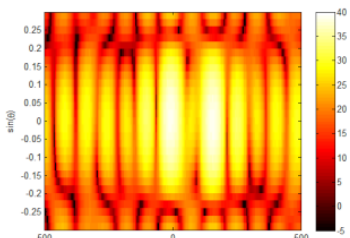

$d=2.00$

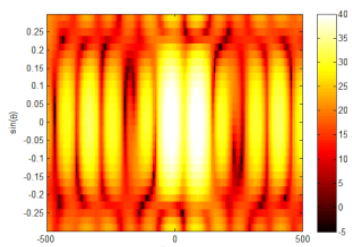

$d \stackrel{\text { Range }}{=} 1.00$

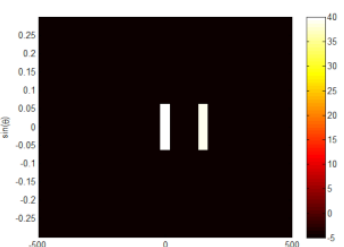

$d \stackrel{\text { Rome }}{=} 2.00$

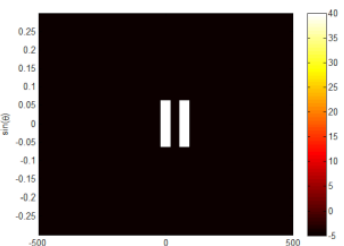

$d \stackrel{\text { Range }}{=} 1.00$

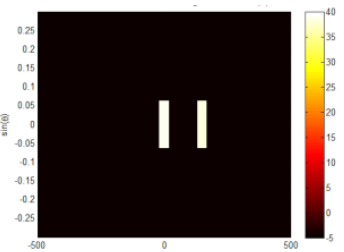

$d \stackrel{\text { Range }}{=} 2.00$

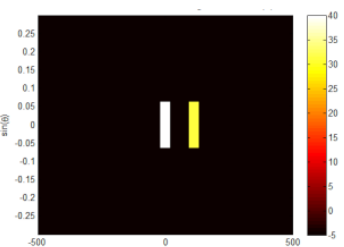

$d=1.00$

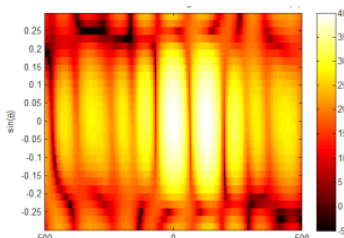

$d \stackrel{\text { Ruge }}{=} 1.75$

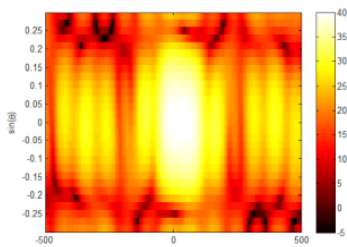

$d \stackrel{\text { Rome }}{=} 0.75$

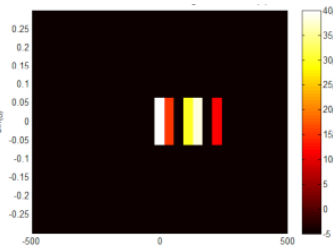

$\stackrel{\text { Range }}{=}=1.75$

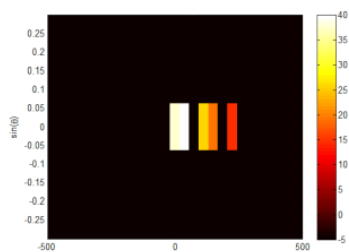

$d \stackrel{\text { Ruge }}{=} 0.75$

(b)

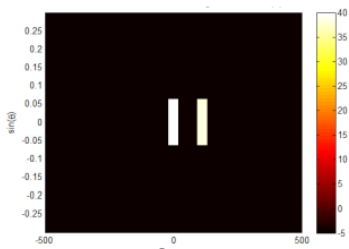

$d=1.75$

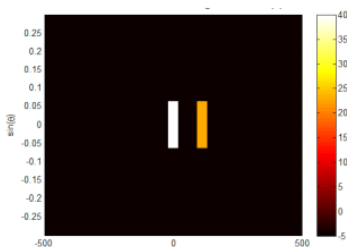

$d=0.75$

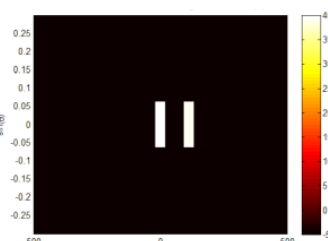

$d \stackrel{\text { Ranpe }}{=} 1.50$

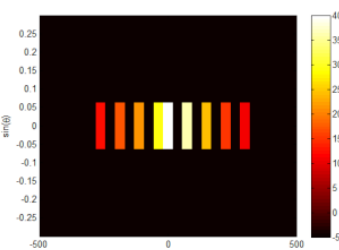

$d=0.50$

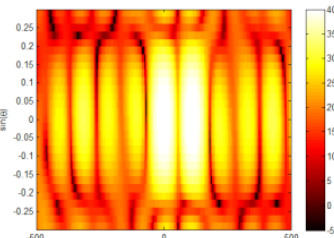

$d \stackrel{\substack{\text { Ruge } \\ d}}{=} 1.50$

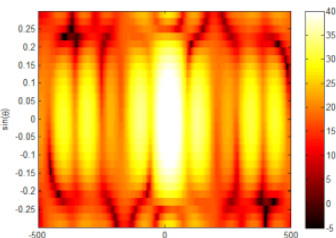

$d=0.50$

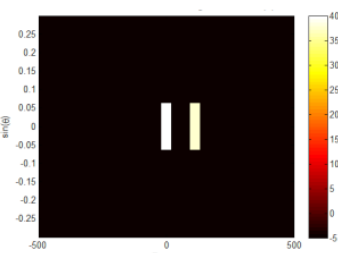

$d \stackrel{\text { Range }}{=} 1.50$

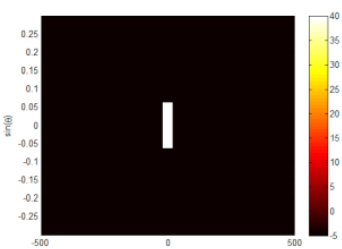

$d=0.50$

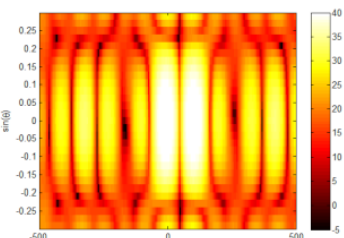

$d \stackrel{\text { Renge }}{=} 1.25$

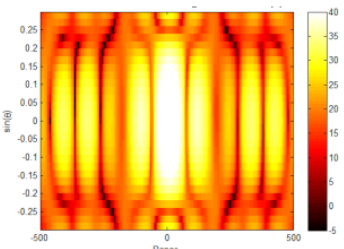

$d=0.25$

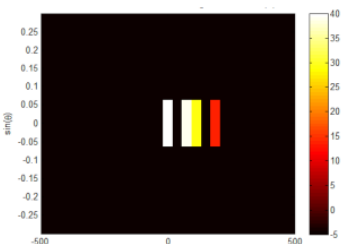

$d \stackrel{\substack{\text { Range } \\=}}{=} 1.25$

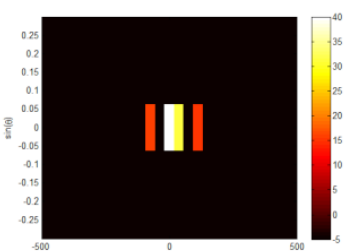

$d=0.25$

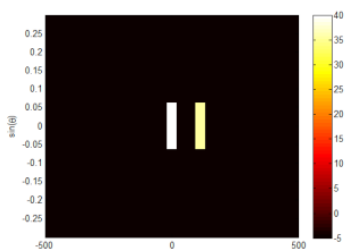

$d=1.25$

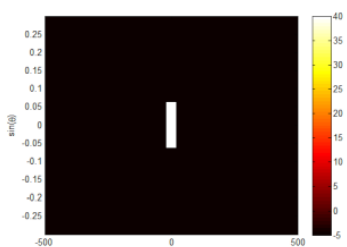

$d=0.25$

(c)

Fig. 5. Outputs for two targets of SNR $=40 \mathrm{~dB}$ at different distances $d$ (in number of range cell widths). Range resolution cell width: $75 \mathrm{~m}$. Angle resolution cell width: 0.25

a Matched filter

b Classical OMP

c Extended OMP 


\section{Results with simulations}

We will now introduce different scenarios in order to exhibit the improvement of robustness with respect to the localisation error. In this section, we consider that all simulated signals fulfil the condition of signal model matching. In other words, we consider that the received signal does not differ from the expected expression of $(10)$.

\subsection{Simulation of two targets at different close distances}

In order to test the "side effect" of the extended rejection method, i.e. how the filter manages two neighbour targets at around one resolution cell width of distance, we define a particular scenario of two targets (same SNR of $40 \mathrm{~dB}$ ) with a decreasing distance $d$ from 2 to 0.25 with steps of 0.25 range cell distances. For the OMP detection steps, we consider a target grid made of two nodes per cell dimension. The left-hand target is fixed on a grid node, while the right-hand target has a changing position, localised on a node only when its distance to the left target is a multiple of 0.5 cell width. To make the comparison, we calculate for each scenario the output of the matched filter, the results after classical OMP and the results after the OMP with extended rejection area. These results are displayed in Figs. 5(a), 5(b), and 5(c), respectively.

Several interesting points of analysis can be deduced from the non-ideal situations: with the classical OMP, several ghost targets are visible each time one target is situated between two nodes of the grid [i.e. for $d \in\{1.75,1.25,0.75,0.25\}]$ or when neighbour target influence occurs [i.e. for $d \in\{0.75,0.5,0.25\}]$. Nevertheless, none of these situations induces any ghost target with the extended OMP. Besides, note two close targets $(d<1)$ are attributed to the same resolution cell, but is not a special issue since within the resolution bound.

Eventually, we have shown that for these particular scenarios, the proposed extension for the OMP rejection step correctly solves the issues of grid granularity and neighbour target influence.

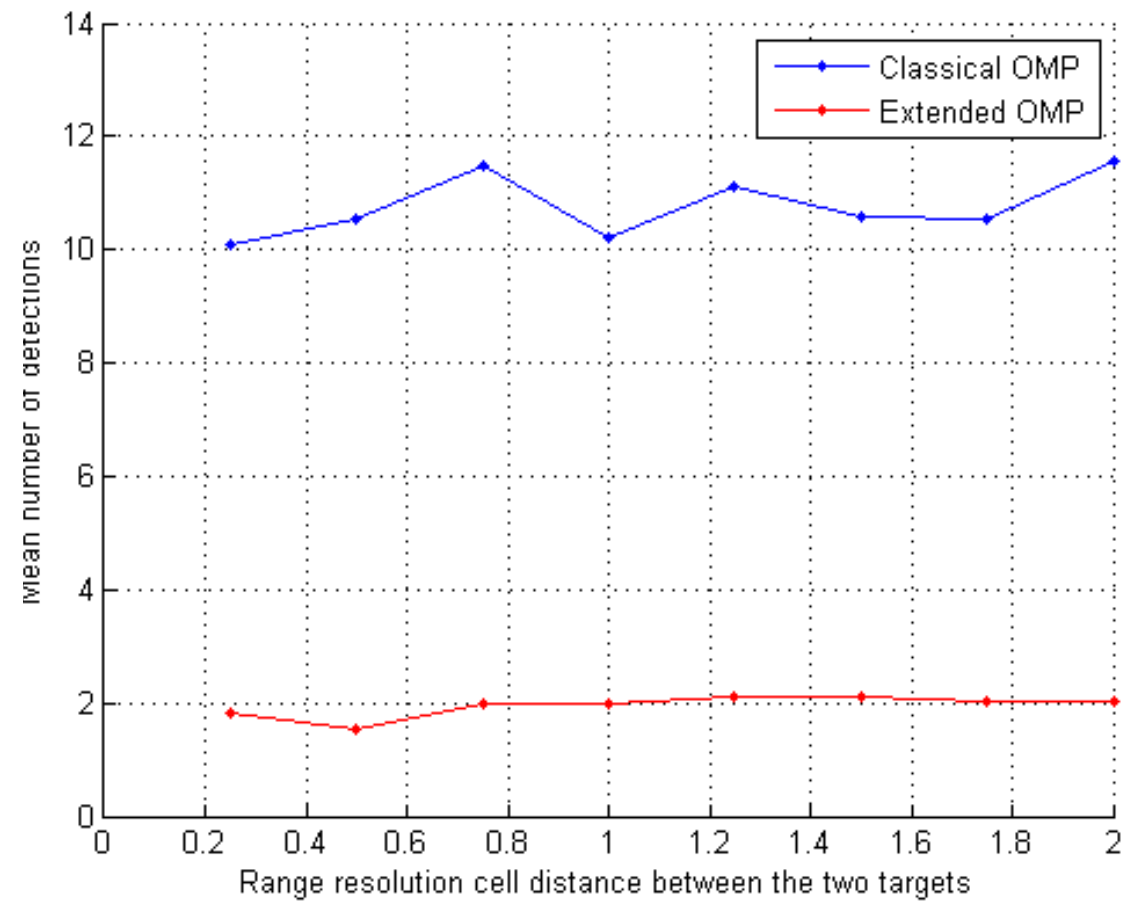

Fig. 6. Average number of targets detected for Monte Carlo simulations of two targets randomly positioned 


\subsection{Performance analysis based on Monte Carlo simulations}

In order to address more general performance consideration, we have chosen to process Monte Carlo simulations with two targets of $40 \mathrm{~dB}$ of SNR positioned randomly on the target space, independently from the grid, and for both classical and extended OMP method applications. We set up different range distances between the two targets, all comprised between 0.25 and 2 range resolution cells. We processed at least 30 runs for each value of the targets separation distance (measured in range resolution cells). Each new run induced a new generation of the noise and of the target positions. We set the detection threshold to the same value as the previous simulation, i.e. $10 \mathrm{~dB}$ above noise level, and for each run, we saved the final number of detected targets, which is actually the total number of real targets plus false alarms.

The results of these simulations are given on Fig. 6. One clearly notices that the classical OMP method always provides many false alarms (around eight in average), while the extended OMP method virtually avoids all false alarms. This is valid for all the target separation distances tested between 0.25 and 2 range resolution cells.

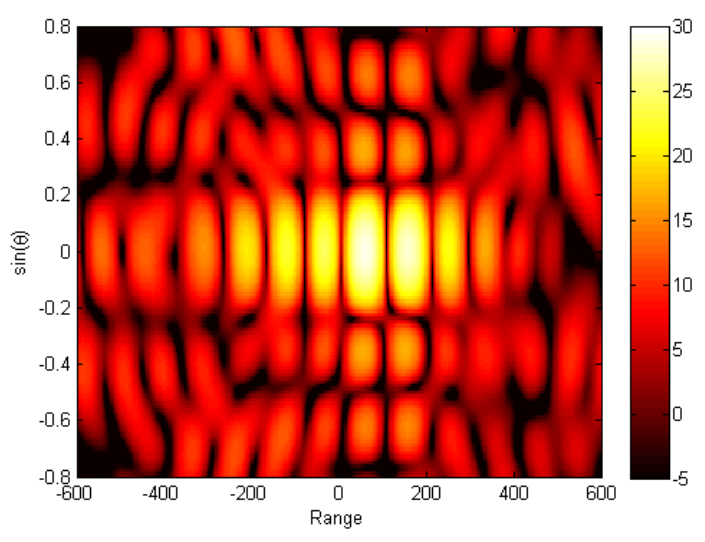

(a)

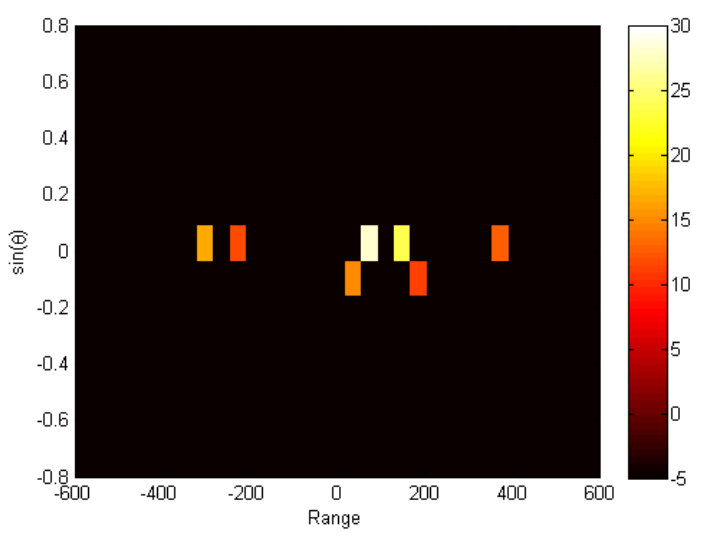

(c)

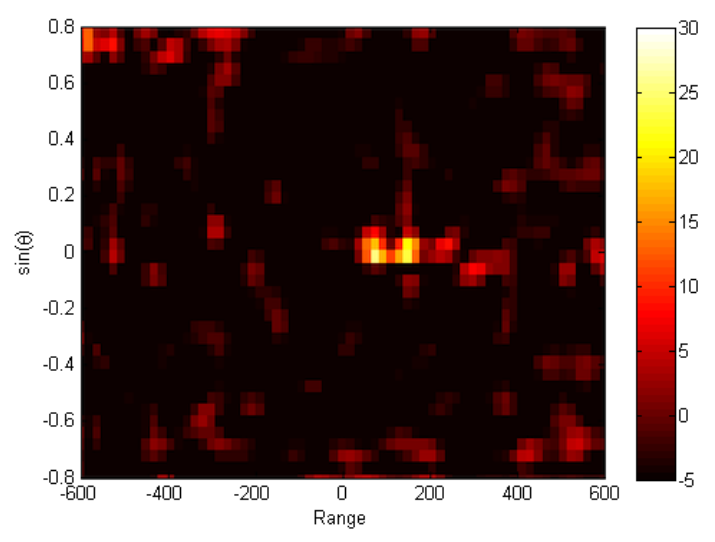

(b)

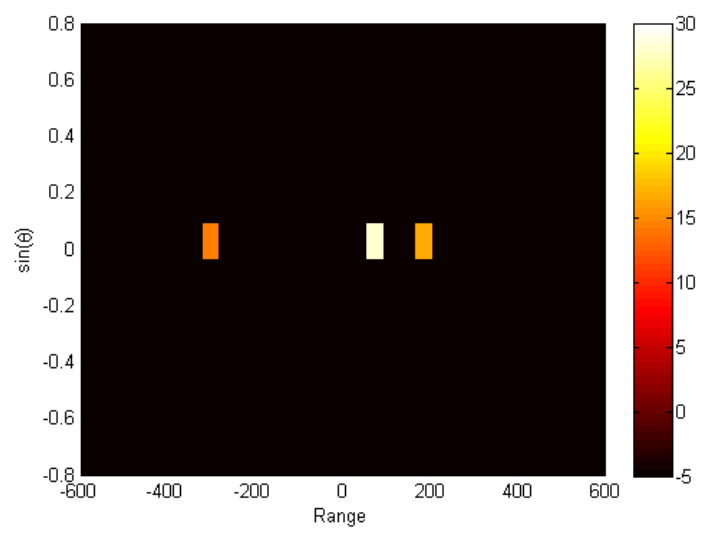

(d)

Fig. 7. Application of the different methods on the experimental data. Range resolution cell width: $75 \mathrm{~m}$. Angle resolution cell width: 0.25

a Matched filter

b IAA (iteration 7)

c OMP - classical rejection

d OMP - extended rejection 


\section{Results with experiments on a real MIMO radar}

We had the opportunity to test the methods introduced in this work on MIMO radar signals acquired in real conditions, thanks to the Hycam platform. This testbed is multifunction S-band radar platform built at ONERA and equipped with 12 independent columns as Tx phased array and 16 columns as Rx antenna [22]. In the experimental scenario, we have generated a family of 12 Gold code waveforms and transmitted them in the Hycam radar in closed-loop configuration. In such configuration, the radiofrequency (RF) circuit arriving at the antennas is bypassed and connected back to the reception chain. The signals are consequently subject to the hardware imperfections of the radar chain and addition of thermal noise from the components. By the way, we have noticed that, in addition of the experimental closed-loop, there is one shorter (unwanted) closed loop which is due to a lack of isolation of the carrier frequency mixer. Therefore, two targets are expected to be detected at short range (order of the hundred of metres) and angle of $0^{\circ}$. Extended description of the signal generation and processing is given in [6] and [23].

Finally, we have applied the target detection methods described in this work on the experimental signals: the matched filter as standard method, the IAA as accurate (but long to compute) method, the classical OMP, and the extended OMP. The resulting outputs are given on Fig. 7. First, one can clearly notice from the ambiguity function (matched filter) of Fig. 7(a) two close mainlobes, so we can deduce they correspond to the two expected "targets" (i.e. the two closed loops). Here, we cannot say if there are other targets since other potential main lobes are impossible to differentiate from the sidelobes. Now, the IAA output of Fig. 7(b) gives two detections, as expected: one at $140 \mathrm{~m}$ corresponding to the radar chain closed loop and one at $80 \mathrm{~m}$ corresponding to the unwanted closed loop. Moreover, for a detection threshold also set at $10 \mathrm{~dB}$, IAA does not give additional detection. Here again, these accurate results are at the price of an extensive computation. Then, the classical OMP results of Fig. 7(c) exhibit the two expected targets plus numerous "ghost" targets, while the extended OMP (Fig. 7(d)) gives the two expected targets plus another one of 14 $\mathrm{dB}(13 \mathrm{~dB}$ below the strongest peak). We assume that this latter detection is a false alarm due to signal model mismatch caused by hardware imperfections, since undetected with the IAA. Besides, note that the right-hand expected target is of lower detected power than with the IAA: indeed, this target is at around one range cell distance to the left-hand target and so is subject to the "side effect" of the extended rejection area.

\section{Model mismatch considerations}

It appeared that although the proposed approach efficiently mitigates model mismatch due to off-grid target location and target proximity, it is still sensitive to model mismatch arising from hardware imperfections. One way to robustify the OMP against these kinds of mismatch would be to extend our approach to any kind of model mismatch, considering now a stochastic target model rather than a deterministic one

$$
z=\mathbf{t}+\mathbf{n} \quad \text { with }\left\{\begin{array}{l}
\mathbf{t} \sim \mathcal{C N}\left(0, \sigma^{2} \mathbf{S}\left(\boldsymbol{\theta}_{0}\right)\right) \\
\mathbf{n} \sim \mathcal{C N}(0, \mathbf{I d})
\end{array}\right.
$$

The term $\sigma^{2}$ is the unknown target power. The term $\mathbf{S}\left(\boldsymbol{\theta}_{0}\right)$, with $\boldsymbol{\theta}_{0}=\left(r_{0}, \theta_{0}\right)$ vector of parameters, is the known normalised power covariance matrix of the target, according to all the (small) variants $\mathbf{a}_{q}$ in the model. It can be decomposed in singular values $\mathbf{v}_{j}$

$$
\begin{aligned}
\mathbf{S}\left(\boldsymbol{\theta}_{0}\right) & =\frac{1}{Q} \sum_{q=1}^{Q} \mathbf{a}_{q}\left(\boldsymbol{\theta}_{0}\right) \mathbf{a}_{q}^{H}\left(\boldsymbol{\theta}_{0}\right) \\
& =\sum_{j} \lambda_{j} \mathbf{v}_{j} \mathbf{v}_{j}^{H}
\end{aligned}
$$


We expect the $\lambda_{j}$ to decrease very quickly in case of small reasonable mismatch. Then we can assume that $\mathbf{v}_{1}=\mathbf{a}\left(\boldsymbol{\theta}_{0}\right)$ in the detection step of the OMP, and use only a few $J$ terms for the rejection step to ensure good cancellation to any variant of the target return. The matrix $\mathbf{A}_{k}$ will hence be written as

$$
\mathbf{A}_{k}=\left[\mathbf{A}_{k-1}, \mathbf{v}_{1}, \mathbf{v}_{2}, \cdots, \mathbf{v}_{j}, \cdots, \mathbf{v}_{J}\right]
$$

Note however that the computation of the projection of the signal onto the $\left(\mathbf{v}_{j}\right)_{j=1 \ldots J}$ will lead to extra computational burden because the $\left(\mathbf{v}_{j}\right)_{j=1 \ldots J}$ will not have good shapes anymore (such as complex exponential with linear phase for instance, leading to computation of the projections with fast Fourier transform (FFT)).

Finally, we can underline that the case of the previous off-grid mismatch is a specific case of model mismatched, where the normalised covariance matrix of the target in (27) can be written as (with $\Delta r, \Delta \theta$ denoting the extension of one resolution cell)

$$
\mathbf{S}\left(r_{0}, \theta_{0}\right)=\frac{1}{\Delta r \Delta \theta} \int_{r_{0}-\frac{\Delta r}{2}}^{r_{0}+\frac{\Delta r}{2}} \int_{\theta_{0}-\frac{\Delta \theta}{2}}^{\theta_{0}+\frac{\Delta \theta}{2}} \mathbf{a}(r, \theta) \mathbf{a}^{H}(r, \theta) d r d \theta
$$

\section{Conclusion}

The use of non-perfectly orthogonal waveforms in MIMO radar systems leads to possible harmful effects. As an illustration, the matched filter applied on phase codes, which gives good target resolution performance, induces a significant "cross-correlation noise" that is likely to make secondary targets impossible to detect. This work has consisted in studying methods that could mitigate the high-level sidelobes while preserving relatively low computational load and a reduced greed on training data. Capon-like methods are efficient but usually need an unrealistic number of training data. The IAA solves this issue but at the price of a high computational complexity. Eventually, we have introduced the OMP, which aims at detecting and rejecting each target contribution, successively. This technique is efficient at condition of correct localisation estimation; but in practice, the influence of neighbour targets and the granularity of the target parameter grid lead to localisation error. We have shown that this issue causes undesired residues and therefore possible false alarms in detected targets. After developments in Taylor series, we have proposed to approximate the real localisation of the target with the detected node, and the eight direct neighbours as well. We have shown that it opportunely widens the rejection area to the resolution cell size. We have applied the proposed method on different scenarios of simulated MIMO radar waveforms, so to prove it has become fully robust with respect to target localisation errors. Moreover, contrary to most of the peak fitting methods, the extended OMP has shown to cope with closely spaced targets. Finally, we have applied the IAA, the classical OMP, and the extended OMP on simulated and then real MIMO radar signals subject to hardware imperfections. We could illustrate that the extended OMP is much faster to compute than the IAA.

In brief, we can say that the extended rejection that we propose for the OMP gives particularly good results for fast detection in the MIMO radar context, at the price of a loss in resolution, though. The sensitivity to signal model mismatch due to hardware imperfections, even if timely reduced, is also still existing. This sensitivity could be mitigated by extending the target a priori spanned subspace to channels errors, in the same way as we have proceeded for the biased target localisation issue. 


\section{References}

[1] Li, J., Stoica, P.: 'MIMO radar-diversity means superiority'. Proc. 14th Adaptive Sensor Array Processing Workshop, 2006, pp. 1-6

[2] Li, J., Stoica, P.: 'MIMO radar with colocated antennas'. IEEE Signal Process. Mag., Sept. 2007, vol. 24, no. 5, pp. 106-114

[3] Chen, C.Y., P.P. Vaidyanathan, P.P.: 'MIMO radar ambiguity properties and optimization using frequency-hopping waveforms'. IEEE Trans. Signal Process., Dec. 2008, vol. 56, no. 12, pp. 59265936

[4] Abramovich, Y., Frazer, G.J., Johnson, B.A.: 'Noncausal adaptive spatial clutter mitigation in monostatic MIMO radar: fundamental limitations'. IEEE J. Sel. Top. Signal Process., Feb. 2010, vol. 4, no. 1, pp. $40-54$

[5] De Maio, A, Lops, M.: 'Design principles of MIMO radar detectors'. IEEE Trans. Aerosp. Electron. Syst., July 2007, vol. 43, no. 3, pp. 886-898

[6] Cattenoz, M., Brouard, P., Brun, A., et al.: 'Coherent collocated MIMO radar: a study on real data'. 14th Int. Radar Symp., Dresden, June 2013, vol. 1, pp. 83-88

[7] Li, J., Stoica, P.: 'Nonparametric adaptive techniques for parameter estimation', in: 'MIMO radar signal processing' (Wiley, 2008), pp. 11-27

[8] Rabaste, O., Savy, L., Cattenoz, M., et al.: 'Signal waveforms and range/angle coupling in coherent colocated MIMO radar'. Int. Conf. on Radar, Sept. 2013, pp. 157-162

[9] Gold, R.: 'Optimal binary sequences for spread spectrum multiplexing'. IEEE Trans. Inf. Theory, Oct. 1967, vol. 13, no. 4, pp. 619-621

[10] Cattenoz, M., Marcos, S.: 'Adaptive processing for MIMO radar realistic non perfectly orthogonal waveforms'. IEEE Radar Conf., Cincinnati, May 2014, pp. 1323-1328

[11] Cattenoz, M., Savy, L., Marcos, S.: 'Adaptive processing methods for MIMO radar experimental signals'. Int. Radar Conf., Lille, Oct. 2014, pp. 1-6

[12] Blunt, S. D., Gerlach, K.: 'Adaptive pulse compression via MMSE estimation'. IEEE Trans. Aerosp. Electron. Sys., Jan. 2006, vol. 42, no. 2, pp. 572-584

[13] Yardibi, T., Li, J., Stoica, P., et al.: 'Source localization and sensing: a nonparametric iterative approach based on weighted least squares'. IEEE Trans. Aerosp. Electron. Syst., Jan. 2010, vol. 46, no. 1, pp. $425-443$

[14] Strohmer, T., Friedlander, B.: 'Compressed sensing for MIMO radar-algorithms and performance'. Asilomar Conf. on Signals, Systems and Computers, 2009, pp. 464-468

[15] Donoho, D. L.: 'Compressed sensing'. IEEE Trans. Inf. Theory, 2006, vol. 52, no. 4, pp. 464-468

[16] Mallat, S., Zhang, Z.: 'Matching pursuit with time-frequency dictionaries'. IEEE Trans. on Signal Process., Dec. 1993, vol. 41, no. 12, pp. 3397-3415

[17] Davis, G., Mallat, S., Zhang, Z.: 'Adaptive time-frequency approximations with matching pursuits', in Montefusco, L., Luigia, P. (Ed.): 'Wavelets: theory, algorithms, and applications' (Academic Press, 1994, Vol. 5), pp. 271-293 
[18] Högbom, J. A.: 'Aperture synthesis with a non-regular distribution of interferometer baselines'. Astronomy and Astrophys. Suppl. Ser., 1974, vol. 15, p. 417

[19] Cornwell, T. J.: 'Högbom's CLEAN algorithm. Impact on astronomy and beyond'. Astron. Astrophys., 2009, vol. 500, no. 1, pp. 65-66

[20] Wiaux, Y., Jacques, L., Puy, G., et al.: 'Compressed sensing imaging techniques for radio interferometry'. Mon. Not. R. Astron. Soc., 2009, vol. 395, no. 3, pp. 1733-1742

[21] Rabaste, O., Savy, L., Desodt, G.: 'Approximate multitarget matched filter for MIMO radar detection via orthogonal matching pursuit'. Int. IEEE Radar Conf., Lille, Oct. 2014, pp. 1-6

[22] Brouard, P., Constancias, L., Brun, A., et al.: 'Hycam: a new S-band surface radar testbed'. IET Int. Radar Conf., Xi'An, Apr. 2013, pp. 1-4

[23] Constancias, L., Cattenoz, M., Brouard, P., et al.: 'Coherent collocated MIMO radar demonstration for air defence applications'. IEEE Radar Conf., Ottawa, Apr. 2013, pp. 1-6 